Revue Française de Civilisation Britannique

\title{
La civilisation économique : interdisciplinarité exemplaire ou limite de l'interdisciplinarité ?
}

Economic Civilisation: Exemplary Interdisciplinarity or the Limits of Interdisciplinary Research?

\section{Vanessa Boullet}

\section{OpenEdition \\ Journals}

Édition électronique

URL : http://journals.openedition.org/rfcb/2917

DOI : $10.4000 /$ rfcb. 2917

ISSN : 2429-4373

Éditeur

CRECIB - Centre de recherche et d'études en civilisation britannique

\section{Référence électronique}

Vanessa Boullet, «La civilisation économique : interdisciplinarité exemplaire ou limite de

l'interdisciplinarité ? », Revue Française de Civilisation Britannique [En ligne], XXIV-1 | 2019, mis en ligne

le 22 mars 2019, consulté le 22 avril 2019. URL : http://journals.openedition.org/rfcb/2917 ; DOI :

$10.4000 / \mathrm{rfcb} .2917$

Ce document a été généré automatiquement le 22 avril 2019

\section{cc) (†) $\odot$}

Revue française de civilisation britannique est mis à disposition selon les termes de la licence Creative Commons Attribution - Pas d'Utilisation Commerciale - Pas de Modification 4.0 International. 


\section{La civilisation économique : interdisciplinarité exemplaire ou limite de l'interdisciplinarité ?}

Economic Civilisation: Exemplary Interdisciplinarity or the Limits of

Interdisciplinary Research?

Vanessa Boullet

\section{Introduction}

1 Tout chercheur commence ses études par l'apprentissage de différentes disciplines. Frédéric Darbellay explique qu' une discipline délimite un domaine de connaissances institutionnalisé dans et par une communauté scientifique donnée, socialement et historiquement située et régie par un paradigme qui définit les présupposés et les objectifs des savoirs à construire $»^{1}$.

Dès son plus jeune âge, l'enfant est confronté à plusieurs disciplines : les mathématiques, le français, l'histoire, pour n'en citer que quelques-unes. Au fur et à mesure des études, les blocs disciplinaires vont se renforcer et des passerelles vont progressivement se mettre en place. Par exemple pour le baccalauréat en France, les lycéens doivent maintenant effectuer un Travail Personnel Encadré, plus communément appelé TPE, en prenant "appui sur une démarche interdisciplinaire», comme l'expliquent les textes ministériels. ${ }^{2}$ Un des objectifs affichés est de: "découvrir les liens qui existent entre les différentes disciplines $»{ }^{3}$ Pourtant, une fois ce lycéen devenu étudiant, il va constater que les études pluridisciplinaires - c'est-à-dire la juxtaposition de plusieurs disciplines sans rompre avec ces dernières - et surtout les études interdisciplinaires - c'est-à-dire une coopération et un dialogue plus ou moins forts entre les différentes disciplines sur un objet d'étude à partir de questionnements communs - demeurent le parent pauvre de l'université, quoiqu'on constate un développement des bi-cursus alliant deux disciplines. La situation est logiquement similaire pour l'enseignant-chercheur. Ce dernier a 
généralement réalisé sa formation universitaire au sein d'une seule discipline et par mimétisme il a logiquement tendance à reproduire ce qu'on lui a enseigné. De plus, il est généralement recruté pour enseigner dans une filière monodisciplinaire. Toutefois, de plus en plus de chercheurs souhaitent mener des recherches ayant des approches pluridisciplinaires ou interdisciplinaires c'est-à-dire qu'ils souhaitent étudier un « objet d'étude complexe » pour reprendre les mots d'Edgar Morin, en "reliant les connaissances » car

3 La prévalence disciplinaire, séparatrice, nous fait perdre l'aptitude à relier, l'aptitude à contextualiser, c'est-à-dire à situer une information ou un savoir dans son contexte naturel. Nous perdons l'aptitude à globaliser, c'est-à-dire à introduire les connaissances dans un ensemble plus ou moins organisé. Or les conditions de toute connaissance pertinente sont justement la contextualisation, la globalisation. ${ }^{4}$

4 Ce type de recherche amène le chercheur à aller au-delà du simple approfondissement de son champ disciplinaire et à essayer de tisser des liens avec d'autres disciplines, d'autres méthodes, afin de mieux comprendre et analyser des phénomènes complexes. On assiste ainsi "à l'émergence d'une pensée en réseau, faite de boucles et de collaborations croissantes $» .^{5}$ Par exemple une approche interdisciplinaire de l'économie permet d'étudier l'interdépendance entre l'économie, la politique et le social ${ }^{6}$. Il permet de prendre plusieurs disciplines des sciences sociales et d'appréhender un système complet et complexe. Le chercheur analyse ainsi comment différentes disciplines interagissent pour initier des changements de paradigmes.

5 Comment le chercheur en civilisation économique peut-il qualifier sa recherche, alors qu'elle se positionne nécessairement dans le dépassement, le débordement des frontières de la discipline pour apporter de la valeur? Est-elle pluridisciplinaire, juxtaposant plusieurs champs d'étude sans les faire interagir? Interdisciplinaire car utilisant les méthodes et les analyses d'une des disciplines pour enrichir un autre champ disciplinaire ? Ou encore transdisciplinaire, dépassant le débat disciplinaire, allant presque jusqu'à créer une nouvelle discipline pour apporter un éclairage novateur. Prend-il alors le risque de constituer un assemblage hasardeux, sans fondement disciplinaire?

\section{La discipline d'origine : l'exemple de la civilisation économique}

6 En 1970, lors de l'introduction de la civilisation dans les études de langues, Roger Raby affirme: "la définition de l'objet des études de civilisation et leurs méthodes restent à inventer, comme l'est d'ailleurs la notion d'humanité ${ }^{7}$. Près de cinquante ans après l'institutionnalisation de la civilisation ${ }^{8}$ dans les études de langue, le débat sur la nécessité de la civilisation s'est tu et Jean-Pierre Fichou explique :

la civilisation double les sciences humaines [il aurait pu ajouter les sciences sociales ou les sciences politiques] en se fondant sur le fait que les étudiants en langues vivantes perdraient beaucoup à faire de l'histoire, de la sociologie ou de l'économie en français et sur la constatation que les instituts qui enseignent ces matières n'offrent que très rarement des cours suffisamment spécialisés sur la société dont ils étudient la langue ${ }^{9}$.

7 Toutefois, la méthodologie de la civilisation reste floue et ses objets imprécis. De plus beaucoup de civilisationnistes gardent toujours une orientation privilégiée qui est souvent historique, sociologique ou politique, plus rarement économique. L'hybridité de 
la civilisation peut donc déconcerter les chercheurs d'autres sciences humaines, sociales, politiques, économiques en France car pour eux, ce pilier de l'enseignement des langues n'est pas une discipline, ou encore les chercheurs étrangers, notamment anglophones, car ils n'arrivent pas à l'assimiler à une discipline équivalente dans leur classification locale. En effet, la civilisation britannique, américaine, irlandaise, espagnole et d'une toute autre aire linguistique, semble "ne pas pouvoir trouver un créneau clair $»^{10} \mathrm{en}$ raison des différentes formes que peut prendre la civilisation selon les centres d'intérêt du chercheur et de l'objet étudié. Est-il envisageable d'étudier un sujet aussi riche et divers que la civilisation selon un prisme unique? Je ne le crois pas, la recherche et l'enseignement de la civilisation doivent «se fonder sur une approche interdisciplinaire à des fins tant d'analyse de l'objet étudié que de transmission du savoir $»^{11}$. La pluridisciplinarité et l'interdisciplinarité qui caractérisent la civilisation, et encore plus la civilisation économique, sont ainsi porteuses d'une connaissance plus étendue, plus transversale, et plus intégrative.

\section{Qu'est-ce ce que la civilisation économique?}

Dans le champ des études de langues, l'étude socio-économique d'un pays constitue un exemple d'enrichissement d'un enseignement "de base " par la pluridisciplinarité et l'interdisciplinarité. En effet, le chercheur tire parti de plusieurs disciplines : il enrichit l'étude des langues par les outils et les méthodes des sciences économiques, des sciences politiques, des sciences sociales (sociologie) et d'autres sciences humaines (l'histoire, la géographie, et parfois la littérature et l'art) pour construire une vision holistique de la civilisation / de la société d'un pays. Le chercheur en civilisation économique étudie ainsi l'économie d'un pays ou d'un ensemble de pays en ayant une approche civilisationnelle, ce que les économistes n'ont pas toujours : il réintègre l'économie au sein du contexte plus large de la politique et de la société d'un pays. Héritier de Fernand Braudel et Karl Polanyi, le chercheur en civilisation économique estime que l'économie d'un pays ne peut pas être comprise hors de son contexte et que les politiques économiques ont besoin d'être reliées à l'histoire et la culture d'un pays ${ }^{12}$. Par exemple, dans ma recherche, j'analyse comment la République d'Irlande est parvenue à changer ses politiques économiques en passant du protectionnisme au libre-échange dans les années 1950. Pour cette étude, il faut tout d'abord comprendre pourquoi Eamon de Valera a souhaité mettre en place un fort protectionnisme en Irlande dans les années 1930: porté par le nationalisme gaélique, il voulait couper tous liens avec le Royaume-Uni, que ce soit politique en réécrivant la constitution irlandaise en 1937 et en transformant l'Irlande en République en 1948, ou tous liens économiques : limiter les échanges économiques avec le Royaume-Uni au strict minimum en créant un état autarcique. Puis il faut comprendre pourquoi il y a une remise en cause de ses politiques dans les années 1950. Pour cela il faut comprendre le contexte mondial après la deuxième guerre mondiale et la mise en place de la mondialisation avec le Plan Marshall: l'Irlande peut bénéficier du Plan Marshall à condition qu'elle ouvre son économie. Il faut également analyser l'état du pays suite aux politiques protectionnistes : la République irlandaise souffre d'une très grave crise économique à la fin des années 1940 et au début des années 1950: aucun gouvernement (malgré une série d'alternance politique) ne parvient à y remédier.

9 Enfin, il faut étudier la conversion des hommes politiques à une nouvelle politique économique en analysant leur cheminement intellectuel, mais aussi leurs discours 
politiques que ce soit devant les Irlandais ou au parlement. Il faut également voir comment apparaissent les nouvelles politiques économiques au sein des économistes puis des hommes politiques, comment elles prennent de l'ampleur et finissent par être adoptées par les différents gouvernements. Enfin, il faut explorer comment ces politiques économiques sont présentées et "vendues » aux Irlandais et également leurs mises en place et leurs conséquences sur la société. Ainsi cette recherche utilise différentes méthodologies et différentes disciplines des sciences sociales, sciences humaines, sciences politiques et sciences économiques: histoire, sciences politiques, analyse du discours, économie, statistiques, etc... Sans cette recherche pluridisciplinaire et interdisciplinaire qui permet la prise en compte du contexte socio-politico-culturel, la compréhension de la politique économique des années 1960 ne peut être totale.

\section{Une mise à l'épreuve?}

10 L'enseignant-chercheur en civilisation et encore plus en civilisation économique ne peut donc pas être rattaché à une seule discipline. Il ne le souhaite probablement pas non plus. Cette position hybride n'est toutefois pas qu'un enrichissement pour le chercheur. C'est également une mise à l'épreuve communicationnelle, épistémologique, méthodologique et institutionnelle car il doit appréhender les méthodes et les concepts de plusieurs disciplines. Il n'est évidemment pas expert en tout, mais il croit qu'il est nécessaire d'étudier le contexte politique, historique, et social afin d'interpréter les faits économiques. Là où certains voient du papillonnage ou une approche superficielle, l'enseignant-chercheur en civilisation voit une prise de recul et au contraire une réflexion systémique et profonde sur un tout complexe. On pourrait presque dire qu'au contraire, c'est l'économiste ou le sociologue qui ne pousse pas l'analyse jusqu'au bout s'il se cantonne uniquement à sa discipline : ni l'un ni l'autre ne peut comprendre tous les facteurs qui ont déclenché le changement radical de la politique économique irlandaise dans les années 1950. Est-ce parce que la situation économique était insupportable? Oui, mais pas seulement, l'économie irlandaise n'en était pas à sa première crise économique. Est-ce grâce à un changement générationnel - version gaëlique de la querelle des Anciens et des Modernes? Oui, mais pas seulement, puisque les sociétés connaissent régulièrement ces changements générationnels. Est-ce parce que la République d'Irlande était prête à tourner la page de son histoire nationaliste et protectionniste ? Oui, mais pas seulement puisque l'Irlande n'a pas pour autant renoncé à ses discours nationalistes. Et ainsi de suite...

11 La même analyse est également possible trente ans plus tard, avec le Tigre Celtique: l'économiste y lira le prolongement logique d'une politique volontariste de développement économique dans un monde acquis au libre-échange, le sociologue y lira l'arrivée à maturité de la génération mieux formée à partir des années 1960, le politologue y verra le succès des politiques tripartites de concertation sociale, l'expert en relations internationales y verra le bilan positif des politiques mises en place avec l'intégration à l'Union Européenne en 1973, et ainsi de suite...

12 Il est donc permis de penser que seul le chercheur qui mène une recherche interdisciplinaire peut appréhender la combinaison de ces différents éléments et en faire la synthèse. Le chercheur qui s'engage dans l'interdisciplinarité vit donc au quotidien une oscillation entre son engagement au service de sa discipline de rattachement (la civilisation) et une saine prise de recul par rapport aux limites disciplinaires, allant 
chercher des méthodologies dans d'autres disciplines, de manière à favoriser sa propre évolution créative. Les chercheurs chahutant les confortables frontières disciplinaires ont pu faire face à de l'incompréhension (« pourquoi mener telle recherche? »), voire même à de la frilosité comme par exemple en 1929 quand Marc Bloch et Lucien Febvre fondent les Annales d'histoire économique et sociale afin de promouvoir l'histoire économique et sociale contemporaine. ${ }^{13}$ En effet, alors qu'au début du siècle, certains historiens ne voyaient pas l'intérêt d'une telle revue, aujourd'hui les Annales sont encensées, mais les difficultés subsistent : par exemple le chercheur peut avoir des difficultés à se former aux autres disciplines : à moins d'avoir le temps de reprendre les études pour avoir une double casquette, le chercheur en civilisation économique ne sera jamais économiste et n'aura jamais un doctorat en économie. En revanche, il n'hésitera pas à dialoguer avec ses collègues économistes. Le chercheur peut également avoir des difficultés à trouver des confrères qui travaillent sur des sujets similaires au sien, ou - malgré Internet - avoir du mal à dialoguer avec les chercheurs de disciplines connexes. Nous sommes en France un petit groupe de civilisationnistes à travailler sur l'économie des pays anglo-saxons. Mais même dans une université multidisciplinaire comme la mienne, les disciplines demeurent cloisonnées, ne serait-ce que par la séparation des campus: les sciences politiques et économiques sont sur un site, les sciences humaines et sociales sur un autre, les sciences dures sur un troisième. Et même au sein des sciences humaines et sociales, sauf à errer de réunion de groupe de recherche en colloques, ce qui nécessiterait beaucoup de temps, il n'est pas aisé de connaitre les chercheurs qui travaillent sur des sujets similaires ou connexes au sien. Par exemple, étant dans un groupe de recherche d'études anglophones, je ne connais pas toujours les recherches de mes collègues spécialistes d'autres langues, ce qui est préjudiciable car cela pourrait engendrer des analyses comparatives très intéressantes. Si cela est difficile au niveau local, cela devient une tâche d'autant plus ardue au niveau national et a fortiori international.

13 Ainsi si la civilisation et peut-être encore plus la civilisation économique est un exemple de pluridisciplinarité et d'interdisciplinarité, cette interdisciplinarité, ce côté « touche-àtout » peut isoler le civilisationniste et rendre sa recherche difficilement visible car il est difficile à caractériser : il n'est ni économiste, ni historien, ni sociologue...

\section{La transdisciplinarité : Se tourner vers les studies}

Ce schéma de production de connaissances interdisciplinaires qui consiste à relier combiner et reformuler des savoirs disciplinaires qui n'avaient pas nécessairement de rapports préalables entre eux est également une marque des studies, qui semblent en mesure d'assumer le choix de l'interdisciplinarité, sans repli disciplinaire ${ }^{14}$. On assiste à une reconfiguration de partages disciplinaires en nouveaux domaines qui se conjuguent au pluriel, comme les gender studies, les cultural studies, les visual studies ${ }^{15} . .$. En effet, à l'inverse des champs scientifiques plus classiques, de nombreux domaines de recherche émergents obéissent à une classification, non pas par discipline, mais par objet d'étude, prenant modèle sur la communauté anglo-saxonne des «studies». Dans la majorité des cas, les «études " relèvent d'une construction thématique et sont donc transversales puisque les études revendiquent leur aptitude à intervenir dans plusieurs disciplines ${ }^{16}$ alors que les disciplines conduiraient à des analyses verticales.

Ainsi quand la recherche sort des sentiers battus, quand il y a un travail transversal entre les disciplines voire au-delà des disciplines, le chercheur ne trouvant pas assez d'espace 
dans sa discipline initiale, pourrait être amené à se tourner vers les domaines des studies qui témoignent de cette volonté de "décloisonnement disciplinaire pour aborder la complexité de problématiques qui ne peuvent être traitées à partir d'une seule discipline» pour citer de nouveau Frédéric Darbellay ${ }^{17}$.

Un chercheur travaillant sur l'Irlande se tournera tout naturellement vers les Irish Studies, une des studies des aires linguistiques, culturelles ou géographiques (area studies). Les Irish Studies couvrent un champ universitaire intégré et pluridisciplinaire, depuis la géographie et les sciences politiques et économiques jusqu'aux études littéraires et historiques, traitant de la variété et de la diversité de l'histoire et de la société irlandaise, du processus complexe par lequel l'identité irlandaise s'est construite. Les universités irlandaises puis anglo-saxonnes, puis européennes ont créé des cursus en études irlandaises, sous la forme de modules multidisciplinaires, permettant aux historiens, sociologues, spécialistes de la langue irlandaise, spécialistes de littérature et autres de coopérer sans $«$ dispute entre disciplines $»^{18}$.

Toutefois, en 2013, des universitaires irlandais déplorent dans un numéro consacré aux Irish Studies qu'avec le temps, l'objectif initial des études irlandaises s'est affaibli et que malgré la multitude des programmes en études irlandaises, la pluridisciplinarité ou l'interdisciplinarité de ces cursus ne s'est jamais traduite en une nouvelle délimitation des frontières disciplinaires ou en une nouvelle organisation des sujets d'étude ${ }^{19}$ : ainsi les spécialistes de littératures demeurent spécialistes de littérature et les historiens, historiens. Au final, les Irish studies, comme beaucoup d'area studies, offrent une ouverture sur d'autres disciplines que le chercheur n'aurait pas forcément activement recherchées, mais la collaboration entre disciplines reste limitée à certains projets et repose au final sur la rencontre et l'affinité, la curiosité et la flexibilité des chercheurs. Ainsi, les Irish Studies sont un facilitateur, permettant des croisements, une ouverture, mais pas de réelle confrontation constructive entre pairs - en tout cas en ce qui concerne l'économie irlandaise. Ce croisement ne se fait pas, peut-être par manque de temps, ou possiblement parce que les Irish Studies embrassent un champ finalement trop large.

Il faut donc trouver des studies plus focalisées, plus thématiques. Ma recherche m'a conduit vers les études de développement ou Development Studies.

Il peut être surprenant de voir une spécialiste de l'Irlande, pays développé, se tourner vers des études qui semblent se focaliser avant tout sur les pays en voie de développement ou les pays émergents. Mais à l'exception de sa position spatiale dans l'hémisphère nord, l'économie irlandaise possédait à la moitié du XXe siècle un très grand nombre de caractéristiques des pays les moins développés de l'hémisphère sud et a d'ailleurs développé les mêmes politiques économiques à cette période. D’ailleurs les économies de l'Europe de l'Est ont aussi rejoint ce champ d'étude. Ainsi, la recherche dans les études du développement, comme pour la civilisation, est très diverse et fonctionne avec d'autres champs de connaissances comme les relations internationales, les global studies (études mondiales), l'économie, les sciences politiques, ou l'environnement. Tout cela crée des opportunités d'enrichissement, d'interactions et de lieux de rencontre pour l'enseignant-chercheur, pour l'étudiant et pour le champ d'étude à part entière. Néanmoins, ce partage peut aussi « affaiblir l'identité du champ d'étude car il devient un espace de médiation («mediated space $»)$ entre les disciplines $»^{20}$.

Finalement, cette recherche pluri- et d'inter-disciplinaire « offre un espace d'incubation à des idées nouvelles par la mise en circulation de concepts $~^{21}$, elle va au-delà des disciplines et étant vecteur d'innovation, peut devenir transdisciplinaire. Frédéric Darbellay définie la 
transdisciplinarité comme : «La transdisciplinarité se définit alors comme une vision globale et intégrée, qui réorganise les savoirs disciplinaires en vue de la compréhension d'un objet d'étude complexe. " ${ }^{22}$ Finalement, aller au-delà des frontières disciplinaires pour comprendre un objet d'étude complexe, voilà ce que fait la civilisation.

L'exemple de ma recherche sur le développement économique de l'Irlande dans la deuxième moitié du vingtième siècle, aboutissant à ce qu'on a nommé le «Tigre celtique ", permet d'illustrer comment plusieurs disciplines peuvent être combinées pour mieux appréhender un sujet. Les économistes verront dans le développement économique irlandais le cas d'un pays qui adopte le libre-échange, attire les investissements directs étrangers et tire parti de ses avantages compétitifs (langue, appartenance à l'Europe etc.). Ils s'appuieront sur des outils statistiques pour démontrer le bienfondé de ce choix. C'est exact, mais cela ne suffit pas à comprendre ni pourquoi ni comment l'Irlande a fait ces choix. L'histoire permet de découvrir le contexte historique de l'Irlande nationaliste du dix-neuvième siècle et la création de l'État libre irlandais puis de la République d'Irlande, et donc d'éclairer la problématique: le passage à une économie ouverte n'était pas une évidence pour un pays naguère protectionniste et nationaliste. L'appel à la sociologie permet d'analyser l'abandon des thèses nationalistes irlandaises, prérequis à l'ouverture sur le monde extérieur. Les artisans de la transformation économique du pays sont parvenus à s'appuyer sur la fierté nationale pour mieux provoquer l'abandon des principes économiques nationalistes. T. K. Whitaker, le haut fonctionnaire à l'origine des plans de développement économique expliquait avoir été frappé par une couverture du magazine satirique, Dublin Opinion, de septembre 1957 où une femme d'âge mûr, symbolisant l'Irlande, consulte une voyante et lui dit "Get to work! They're saying I've no future" ${ }^{23}$. Avec la linguistique et la rhétorique il est possible d'analyser les discours des hommes politiques et la mise en place d'un nouveau vocabulaire, contribuant au renouvellement des théories politiques et économiques tout en préservant le sentiment de fierté nationale. Les sciences politiques enfin permettent d'analyser l'alternance politique qui se met en place, l'arrivée d'une nouvelle génération au pouvoir ayant une conception différente de l'Irlande. Par exemple, Sean Lemass, ministre de l'Industrie et futur Premier ministre, pendant une convalescence, décline la suggestion d'Eamon de Valera d'améliorer son gaélique mais demande à T.K. Whitaker de lui conseiller des ouvrages économiques ${ }^{24}$. Seule la combinaison de ces disciplines permet de comprendre la richesse de la problématique, de l'analyser et de l'expliquer.

\section{Vers des équipes de recherche interdisciplinaires?}

Ceci étant dit, il conviendrait de m'interroger sur ma propre pratique de la très interdisciplinaire civilisation économique et sur les nécessaires limites d'un unique chercheur qui prétend utiliser plusieurs outils, chacun d'entre eux étant intrinsèquement complexe. Le fait est que je ne suis ni économiste, ni sociologue, ni historienne. Pourtant je pense enrichir la réflexion de l'économiste qui analyse le développement de l'économie irlandaise, car le seul point de vue économique ne suffirait probablement pas à expliquer pourquoi les Irlandais ont accepté une politique de modération salariale dans les années 1980 ou après la crise économique de 2008, pourtant les économistes s'accordent sur le fait que cette attitude a fortement contribué au succès de l'économie irlandaise. Je complète le point de vue du sociologue qui pourrait constater la fierté des Irlandais face aux succès du Tigre Celtique, mais peut-être pas complètement appréhender comment les 
Irlandais se sont appropriés les politiques économiques qui au cours de plusieurs décennies ont préparé ce succès. Enfin, l'historien seul ne pourrait que constater avec étonnement que l'Irlande tourne aussi facilement la page de décennies de protectionnisme pour adopter le libre-échange dans les années 1960. L'apport du civilisationniste n'est évidemment qu'incrémental, mais cela ne veut pas dire pour autant qu'il est anecdotique.

Pour autant, bien que je me sois formée, parfois non sans difficultés à certains outils notamment économiques ou statistiques - peu de professeurs de langues comprennent sans explication mes graphiques à l'échelle semi-logarithmiques, alors qu'ils semblent triviaux aux économistes - je dois admettre que j'ai certainement fait des approximations voire des erreurs, bien que j'espère avoir corrigé la plupart d'entre elles de moi-même ou lors d'une relecture par un confrère suffisamment curieux pour s'intéresser à l'approche «différente» que j'affectionne. Ces approximations sont-elles dommageables? Peut-être, mais gardons en tête humblement que la connaissance humaine se construit par essais et erreurs. Sont-elles regrettables? Sans aucun doute. Mais comment les éviter?

Malheureusement, malgré les colloques, la recherche reste très structurée par discipline et donc assez solitaire pour le civilisationniste. J'appelle de mes vœux la création de groupes de recherches véritablement pluri et interdisciplinaires en sciences humaines, politiques et économiques, dans lesquels les spécialistes de plusieurs disciplines puissent s'enrichir mutuellement, accepter les erreurs de leurs collègues et les corriger, s'enrichissant par la réciprocité.

\section{Conclusion}

Tout jeune chercheur a besoin pour débuter sa recherche d'un ancrage disciplinaire : c'est un préalable essentiel à la réflexion, mais également à la structuration et à la circulation des idées, et aussi un facteur déterminant de leur réception. Sans les disciplines, le chercheur ne peut ébranler le savoir, le déconstruire pour le reconstruire. Ce besoin de disciplines n'empêche pas une interaction heuristique et féconde avec les champs disciplinaires connexes ou plus éloignés, tendant ainsi dans un premier temps vers la pluridisciplinarité puis l'interdisciplinarité quand le chercheur maîtrise les concepts et les théories pour pouvoir ainsi transposer un concept ou une méthode d'un champ d'étude à une autre. Le chercheur qui serait tenté par cette transversalité finira probablement par se sentir à l'étroit ou isolé dans son champ disciplinaire d'origine et pourra se tourner vers les Studies. Mais même au sein des études, l'interdisciplinarité et la transdisciplinarité ne sont pas toujours présentes, ou du moins certains domaines trouveront mieux leur place dans certaines études. C'est donc au chercheur de trouver le lieu qui convient le mieux à sa recherche, lequel peut évoluer dans le temps. Le chercheur pourrait ainsi passer de sa discipline aux studies géographiques puis aux studies thématiques. Cette oscillation et collaboration entre les différents lieux et interactions de savoirs ne remet pas en cause les fondements épistémologiques des disciplines. Elle repose même sur une interaction féconde entre lieux de recherche, comme l'interdisciplinarité l'est pour la recherche.

Cette hybridité est également importante pour l'enseignement : les étudiants ont besoin des fondamentaux apportés par les disciplines « classiques », et de plus en plus de cursus 
prônent la pluridisciplinarité. Une fois les bases en place, les étudiants peuvent appréhender la pluridisciplinarité, voire l'interdisciplinarité. En effet, l'internationalisation et l'interconnexion croissante des économies, des cultures et des sociétés exigent de plus en plus des compétences de plusieurs disciplines comme l'étude des sciences économiques, et des langues étrangères. Mes étudiants de langues étrangères appliquées croisent les sciences économiques et les langues étrangères, mais ils n'arrivent toujours pas aisément à mener une analyse interdisciplinaire, généralement par manque de recul sur l'objet d'étude, par manque de mobilisation des savoirs et par manque de pratique à établir des passerelles entre les disciplines et à formuler des questionnements et des problématiques communs.

Les disciplines sont donc toujours présentes dans nos recherches, mais au lieu d'être l'unique point d'ancrage, elles peuvent être l'indispensable point de départ d'un parcours. Elles apportent un cadre méthodologique et théorique, que l'étudiant puis le chercheur, enclin à l'interdisciplinarité ou à la transdisciplinarité, habité par une ardeur intellectuelle, voire une certaine témérité, dépassera sans les abandonner, pour échanger, collaborer, et innover.

Vanessa BOULLET est Maître de conférences en LEA à l'Université de Lorraine (Nancy). Sa recherche porte sur la civilisation irlandaise, plus particulièrement sur les interactions entre économie, société et politique. Elle a co-dirigé avec Claudine Armand et David Ten Eyck un livre sur l'interdisciplinarité : Enjeux et positionnements de l'interdisciplinarité (P.U. Nancy, 2014).

\section{BIBLIOGRAPHIE}

BURGUIERE, André, « Histoire d'une histoire : la naissance des Annales », Annales. Économies, Sociétés, Civilisations, nº 6 (1979), pp. 1347-1359.

COSSU-BEAUMONT, Laurence, Coste, Jacques-Henri et Velut, Jean-Baptiste (dir.), The Crisis and Renewal of U.S. Capitalism: A Civilizational Approach to Modern American Political Economy (Londres, Routledge, 2015).

CRONIN, Michael G. et Nolan, Emer, "Ideas and Institutions after the Crash", The Irish Review, No. 46 (Autumn 2013), pp. 1-3.

CURRIE-ALDER, Bruce, "The state of development studies: origins, evolution and prospects", Canadian Journal of Development Studies / Revue canadienne d'études du développement, 37:1 (2016), pp. 5-26.

DARBELLAY, Frédéric, « Où vont les studies ? Interdisciplinarité, transformation disciplinaire et pensée dialogique », Questions de communication, 25 (2014), pp. 173-186.

DARBELLAY, Frédéric, « Vers une théorie de l'interdisciplinarité? Entre unité et diversité », Nouvelles perspectives en sciences sociales : revue internationale de systémique complexe et d'études relationnelles, vol. 7, n 1 (2011), pp. 65-87. 
DARBELLAY, Frédéric, « Préface ", in Claudine Armand, Vanessa Boullet et David Ten Eyck (dir.), Enjeux et positionnements de l'interdisciplinarité (Nancy, PUN, 2014), pp. 7-12.

EDUSCOL, « Présentation des travaux personnels encadrés et des thèmes nationaux », http:// eduscol.education.fr/cid47789/tpe.html, consulté le 6 juillet 2018.

FICHOU, Jean-Pierre, Enseigner les civilisation (Paris, PUF, coll. L'éducateur, 1979).

LE MOËL, Sylvie \& DE NERVAUX-GAVOTY, Laure (dir.), De l'interdisciplinarité à la transdisciplinarité (Berne, Peter Lang, 2016).

MORIN, Edgar, « Réforme de pensée, transdisciplinarité, réforme de l'Université », http://cirettransdisciplinarity.org/bulletin/b12c1.php, consulté le 6 juillet 2018.

PEYRONEL, Valérie , "L'enseignement de la civilization au Coeur de l'approche interdisciplinaire: le cas de l'Irlande du Nord, in Claudine Armand, Vanessa Boullet et David Ten Eyck (dir.), Enjeux et positionnements de l'interdisciplinarité (Nancy, PUN, 2014), pp. 69-86.

\section{NOTES}

1. Frédéric Darbellay, « Où vont les studies ? Interdisciplinarité, transformation disciplinaire et pensée dialogique », Questions de communication, 25, 2014.

2. Eduscol, "Présentation des travaux personnels encadrés et des thèmes nationaux ", http:// eduscol.education.fr/cid47789/tpe.html, consulté le 6 juillet 2018.

3. Ibid.

4. Edgar Morin, « Réforme de pensée, transdisciplinarité, réforme de l'Université », http://cirettransdisciplinarity.org/bulletin/b12c1.php, consulté le 6 juillet 2018.

5. Sylvie Le Moël et Laure de Nervaux-Gavoty, De l'interdisciplinarité à la transdisciplinarité (Berne, Peter Lang, 2016), p. 214.

6. Laurence Cossu-Beaumont, Jacques-Henri Coste et Jean-Baptiste Velut, The Crisis and Renewal of U.S. Capitalism: A Civilizational Approach to Modern American Political Economy (Londres, Routledge, 2015), p. 4.

7. Jean-Pierre Fichou, Enseigner les civilisations (Paris, PUF, coll. L'éducateur, 1979), p. 10.

8. L'épreuve de civilisation est à l'agrégation de langues en 1977 pour la première fois.

9. Jean-Pierre Fichou, op. cit., p. 10.

10. Valérie Peyronel, "L'enseignement de la civilization au Coeur de l'approche interdisciplinaire: le cas de l'Irlande du Nord, in Claudine Armand, Vanessa Boullet et David Ten Eyck (dir.), Enjeux et positionnements de l'interdisciplinarité (Nancy, PUN, 2014), p. 70.

11. Ibid.

12. Laurence Cossu-Beaumont, Jacques-Henri Coste et Jean-Baptiste Velut, op. cit., p. 4

13. André Burguière. "Histoire d'une histoire : la naissance des Annales", Annales. Économies, Sociétés, Civilisations. $\mathrm{N}^{\circ}$ 6, 1979. pp. 1347-1359.

14. Frédéric Darbellay, op. cit., p. 4.

15. Claudine Armand, Vanessa Boullet et David Ten Eyck (dir.), Enjeux et positionnements de l'interdisciplinarité (Nancy, PUN, 2014), p. 7.

16. Comme les études culturelles, les études de genre, les animal studies, les environmental studies, etc.).

17. Frédéric Darbellay, op. cit., p. 4.

18. Michael G. Cronin et Emer Nolan, "Ideas and Institutions after the Crash", The Irish Review, No.

46, Autumn 2013, p. 2.

19. Ibid. 
20. Bruce Currie-Alder, "The state of development studies: origins, evolution and prospects", Canadian Journal of Development Studies / Revue canadienne d'études du développement, 37:1, 2016, pp. 5.

21. Frédéric Darbellay, «Préface », in Claudine Armand, Vanessa Boullet et David Ten Eyck (dir.), Enjeux et positionnements de l'interdisciplinarité (Nancy, PUN, 2014), p. 9.

22. Frédéric Darbellay, « Vers une théorie de l'interdisciplinarité? Entre unité et diversité ", Nouvelles perspectives en sciences sociales : revue internationale de systémique complexe et d'études relationnelles, vol. 7, n 1, 2011, pp. 75-76.

23. Entretien avec T. K. WHITAKER, 13 juillet 2004

24. Quand Lemass tombe malade au milieu des années 1950, de Valera vient lui rendre visite, accompagné de Whitaker

Whitaker, T. K.

et lui propose d'utiliser ce temps libre pour améliorer son irlandais. Lemass se tourne alors vers Whitaker et lui demande s'il peut plutôt lui conseiller quelques bons livres d'économie. Lemass est l'un des rares ministres qui maîtrise les théories économiques à cette époque. Martin Mansergh, “The Political Legacy of Seán Lemass”, Études Irlandaises, vol. 2, 2000, p. 151.

\section{RÉSUMÉS}

Cet article s'interroge sur la place de la recherche et de l'enseignement interdisciplinaire au travers de l'exemple de la civilisation économique. La civilisation cherche à appréhender les différentes facettes d'un fait culturel, d'une évolution sociale ou d'une situation historique. Pour ce faire, elle fait appel à de nombreuses disciplines et méthodes (l'histoire, la géographie, les sciences sociales et politiques pour n'en citer que quelques-unes). Elle est donc essentiellement interdisciplinaire et ne peut pas être affiliée à une discipline clairement définie (comme l'histoire, la sociologie ou l'économie). C'est peut-être pour cette raison que son image est parfois ternie par un manque de reconnaissance scientifique et que la recherche et les enseignements en civilisation laissent perplexes nos collègues étrangers qui appréhendent difficilement le sens que nous donnons au terme « civilisation ».

Quand en plus de la civilisation, le chercheur traite d'économie, comment peut-il qualifier sa recherche, alors qu'elle se positionne nécessairement dans le dépassement, le débordement des frontières de la discipline pour apporter de la valeur? Est-elle pluridisciplinaire, juxtaposant plusieurs champs d'étude sans les faire interagir? Interdisciplinaire car utilisant les méthodes et les analyses d'une des disciplines pour enrichir un autre champ disciplinaire ? Ou encore transdisciplinaire, dépassant le débat disciplinaire, allant presque jusqu'à créer une nouvelle discipline pour apporter un éclairage novateur. Prend-elle alors le risque de constituer un assemblage hasardeux, sans fondement disciplinaire?

This paper will analyse the notion of interdisciplinary research and teaching through the lens of economic civilisation. Civilisation research covers the various dimensions of cultural, social or historical events. It refers to and uses a number of disciplines and methods borrowed notably from humanities and social sciences. Civilisation is essentially interdisciplinary and cannot be 
adequately comprehended using only one discipline (such as sociology, history or economics). This might explain why the image of civilisation is sometimes tarnished by a lack of scientific recognition or why non-French lecturers and researchers are often puzzled by lectures in "civilisation". Adding the word 'economics' to form 'economic civilization' does not make it any easier.

How can we define this research area that in essence goes beyond the frontiers of disciplines to create meaning? Is this research pluri-disciplinary in nature, leveraging several fields of studies without combining them? Is it interdisciplinary as, it uses the methods of one or several of the disciplines to enhance another discipline? Or, is it transdisciplinary, going beyond the disciplinary debate to the point of sometimes creating a whole new field of study to shed new light on old questions, but taking the risk of creating a muddled assemblage of ideas without solid foundations?

INDEX

Keywords : civilisation, interdisciplinarity, economics, research, Ireland

Mots-clés : civilisation, interdisciplinarité, économie, recherche, Irlande

\section{AUTEUR}

VANESSA BOULLET

IDEA, Université de Lorraine 